\title{
Frailty in liver transplantation: A comprehensive review
}

\author{
AnnMarie Liapakis ${ }^{1,2}$, (1) Elizabeth Morris ${ }^{1,3}$, (1) Sukru Emre ${ }^{1,4}$ \\ ${ }^{I}$ Yale New Haven Transplantation Center, USA; ${ }^{2}$ Division of Digestive, Department of Internal Medicine, Disease Associate Professor of Medicine and Surgery, \\ Yale University School of Medicine, USA; ${ }^{3}$ Yale New Haven Health, Nutrition Services, USA; ${ }^{4}$ Department of Surgery (retired), Department of Surgery and \\ Pediatrics, Yale University School of Medicine, USA
}

\begin{abstract}
Frailty, a global impairment of multiple organ systems resulting in increased vulnerability to health stressors, is common in end-stage liver disease, multifactorial in etiology, and impacts overall mortality as well as outcomes in liver transplantation. This is a review of the currently available data, a synopsis of expert consensus, and a framework for transplant centers to approach frailty. We suggest that centers use a multidisciplinary team of healthcare providers and approach frailty in a programmatic fashion to provide effective patient care and ensure optimal transplant outcomes. The utilization of standardized protocols to address both malnutrition and physical debility is ideal and can help ensure safety. A toolbox of resources has been made available by experts in the field to facilitate this approach. The incorporation of new technology tailored to overcome barriers is another resource under investigation.
\end{abstract}

Keywords: Frailty; liver transplantation; sarcopenia.

\section{Introduction}

Frailty has been defined as a "global impairment of multiple organ systems resulting in increased vulnerability to health stressors." ${ }^{[1]}$ Frailty is common among patients with end-stage liver disease (ESLD). One recent multicenter study found frailty to be present in $25 \%$ of outpatients. ${ }^{[2]}$ It is even more common among hospitalized patients. ${ }^{[3]}$ The pathogenesis of frailty is multifactorial, and includes hepatic dysfunction, as well as malnutrition, low physical activity level (both of which may be exacerbated by recurrent hospitalization), systemic inflammation, and hypogonadism with resultant sarcopenia. ${ }^{[4]}$ Frailty results in an overall reduced functional capacity and reserve. Both liver dysfunction and frailty impact mortality in patients with ESLD. ${ }^{[5]}$ Frailty contributes to mortality independent of hepatic decompensation. ${ }^{[2]}$ To optimize care, frailty must be formally assessed and addressed in a timely and efficient manner. Expert consensus has noted that poor caloric intake, low physical activity, and muscle depletion are integral components of frailty,

How to cite this article: Liapakis AM, Morris E, Emre S. Frailty in liver transplantation: A comprehensive review. Hepatology Forum 2021; 2(2):80-88.

Received: May 09, 2021; Accepted: May 10, 2021; Available online: May 24, 2021

Corresponding author: AnnMarie Liapakis; PO Box 208019, 333 Cedar Street, LMP 1080, New Haven, CT 06510, USA

Phone: +1 203 737-6890; e-mail: Annmarie.liapakis@yale.edu

(1) O OPEn Access

c. This work is licensed under a Creative Commons Attribution-NonCommercial 4.0 International License.

(C) Copyright 2021 by Hepatology Forum - Available online at www.hepatologyforum.org and represent specific targets for intervention. ${ }^{[4]}$ Standards of practice that provide nutritional support and rehabilitation services are critical. This is particularly important in the context of liver transplantation (LT), where frailty has been shown to impact both pre- and post-transplant outcomes, including liver waitlist removal and death. ${ }^{[6]}$

LT is the goal for patients with ESLD, since there is no other curative therapy available. Frailty in ESLD patients may be present before or develop after a patient is listed for transplant. Providers should focus their clinical practice on the provision of comprehensive care with a broad perspective of "patient" optimization, not simply "liver" care. Providers must assess not only the severity of a patient's liver disease, but also evaluate the operative risk, predicted perioperative course, and potential for long-term survival. Modifiable barriers should be identified and addressed in a proactive fashion to ensure patient success and optimal allocation of scarce liver allografts. Standards of practice that assess frailty and provide for early intervention are essential.

\section{Pathogenesis of Malnutrition and Sarcopenia in ESLD}

Malnutrition has been reported to be present in $50 \%$ to $90 \%$ of patients with ESLD, depending on the assessment methods used. ${ }^{[7]}$ One review found that malnutrition was present in $20 \%$ of patients with compensated cirrhosis and $60 \%$ to $99 \%$ of patients with decompensated cirrhosis. ${ }^{[8]}$ The etiology of malnutrition is multifactorial. This includes a loss of appetite due to dysgeusia, nausea, bloating, and early satiety. Patients with ESLD may also have malabsorption, altered gut motility, and dysbiosis. Intestinal hyperpermeability may lead to further protein loss. These patients also have depleted glycogen stores and increased energy expenditure due to increased catecholamines and increased myostatin, which is a negative regulator of skeletal muscle. Various medications may also impact nutritional status. ${ }^{[9]}$ It is also our experience that the need for recurrent medical interventions, such as regular large-volume paracentesis, which depletes protein stores, and restricted oral intake in the setting of diagnostic and therapeutic procedures, contribute to malnutrition.

The pathogenesis of sarcopenia in ESLD is multifactorial as well. As noted above, nutritional deficiency with a lack of sufficient caloric intake of protein, carbohydrates, and fat, leads to low serum levels of branch chain amino acids (BCAAs). Proinflammatory cytokines are released, which leads to ubiquitin-proteasome degradation. Hyperammonemia leads to myostatin activation, which impairs mitochondrial function, increases muscle autophagy, and elevates elF2 phosphorylation. Loop diuretics utilized in ascites management may lead to impaired skeletal myoblast differentiation. In male patients, hypotestosteronemia may lead to muscle cell apoptosis and myostatin activation. Increased hepatic gluconeogenesis causes an increase in BCAA uptake and metabolism. Patients may have reduced physical activity, which causes muscle 
cell apoptosis and mechanistic target of rapamycin (mTOR) inhibition. Patients with alcoholic cirrhosis may have increased muscle autophagy due to myostatin activation and mTOR inhibition. Patients who are taking glucocorticoids and have impaired insulin and insulin-like growth factor 1 signaling will have ubiquitin-proteasome degradation. ${ }^{[9]}$

\section{Tools for Assessing Frailty}

Sarcopenia can be objectively measured and is a distinct independent predictor of mortality in patients with cirrhosis as well as a contributor to frailty. ${ }^{[10]}$ Various methods may be utilized, as presented in Table 1. Psoas muscle thickness is a validated measure that may be readily obtainable, given that a CT scan is commonly used to screen ESLD patients for hepatocellular carcinoma (HCC). Durand et al. ${ }^{[1]}$ demonstrated that patients with ESLD had a reduced survival rate when psoas muscle thickness was low $(<16.8 \mathrm{~mm} / \mathrm{m})$. Englesbe et al. ${ }^{[12]}$ also reported that the hazard ratio for mortality increased for patients with low psoas muscle thickness $(<16.8 \mathrm{~mm})$. Central sarcopenia has been found to correlate with the severity of frailty. ${ }^{[13]}$

Frailty can also be objectively measured via validated tools described in Table 1. The Liver Frailty Index (LFI), which was designed specifically for patients with liver disease, is one example and may be readily incorporated into clinical care. ${ }^{[14]}$ The LFI measures handgrip strength, chair stands, and balance. LFI assessments of ESLD patients are more likely to be classified as pre-frail or frail than those of healthy individuals, and robust less often than adults without liver disease. The LFI is exceptionally reliable; it has an intraclass correlation coefficient (ICC) of 0.93 (95\% confidence interval: $0.91-0.95)$ and is highly reproducible. ${ }^{[15]}$ The serum sodium modification of the Model for End-Stage Liver Disease-Sodium (MELD-Na) utilizes standard laboratory values; bilirubin, creatinine, and serum sodium levels; and the international normalized ratio to calculate a score measuring liver disease severity. The model also provides an objective 3-month mortality risk assessment, and is used to prioritize liver waitlist candidates for organ allocation. ${ }^{[16-18]}$ However, the MELD-Na score does not incorporate any measurement of frailty. Lai et al. ${ }^{[14]}$ developed the Net Reclassification Index (NRI) to examine patient classification when the LFI score was added to the MELD-Na score. The index correctly reclassified $16 \%$ of deaths/transplant waitlist de-listings $(\mathrm{p}=0.005)$ and $3 \%$ of nondeaths/de-listings $(\mathrm{p}=0.17)$ with a total NRI of $19 \%(\mathrm{p}<0.001)$. This was a better predictor of waitlist mortality than the MELD-Na score alone. The American Society of Transplantation (AST) advocates use of the LFI in the baseline and longitudinal assessment of liver transplant patients. ${ }^{[4]}$

\section{Addressing and Reversing Frailty}

Lai $^{[19]}$ provided a framework to guide LT decision-making, a significant contribution to the understanding of frailty in ESLD. She noted the need to consider conditions that may limit the likelihood of a return to health and recaptured functional status, and that these risks should be evaluated in addition to other factors that prompt the need for LT. A low probability of restoration risks adverse post-transplant outcomes. Futile transplants are to be avoided in order to minimize patient suffering and avoid inappropriate resource allocation. Frail patients (LFI $>4.5$ ) with a prolonged lag in restoration post-transplant are subject to extended intubation, ${ }^{[20]}$ intensive care unit/hospital stays $^{[21]}$ and multiple complications, including infections due to bedbound status, ${ }^{[22]}$ and mortality. ${ }^{[21]}$ We hold that healthcare providers should identify frailty as early as possible and provide comprehensive care using standardized protocols to address both malnutrition and physical debility to provide optimal opportunity for recovery and appropriate decision-making. Frailty is an important consideration when evaluating transplant candidacy and suitability.

Professional societies such as the American Association for the Study of Liver Disease (AASLD) and the AST have highlighted the importance of this. The AASLD outlines three steps that should be taken to address frailty in ESLD. The first step is to inform, i.e., educate, the patient and the multidisciplinary team about the importance of addressing frailty. The second step is to measure frailty, and they note the need for a balance of ease, consideration for the complexity of the issue, and objectivity. The third step is to reverse frailty, which requires a combination of nutrition and physical rehabilitation, i.e., exercise-based interventions. ${ }^{[23]}$ An AST report on frailty in solid organ transplantation noted that frailty is a common problem in patients with end-stage organ disease awaiting transplantation and that it contributes to mortality on the waitlist and in the posttransplant period. While the optimal methods of assessing frailty may vary according to the organ to be transplanted, interventions to address and reverse frailty appear promising. ${ }^{[2]}$

\section{Malnutrition in ESLD: Nutrition Intervention}

Nutritional assessment of a patient draws from both objective and subjective data. Nutritional intake may be assessed using tools such as a dietary recall interview, a food diary, or a calorie count. Hydration status influences body composition and therefore may affect and limit the value of weight and body mass index (BMI) measurements. Instruments include a subjective global assessment $(\mathrm{SGA})^{[25]}$ and the Royal Free Hospital-Nutritional Prioritizing Tool (RFH-NPT). ${ }^{[26]}$ Several methods to evaluate nutritional risk and assess nutritional status are provided in Table 1. Anthropometric testing can also be utilized, including measurement of the triceps skin fold, mid-upper arm circumference, and handgrip strength. Blood test values, such as albumin and prealbumin, are unreliable as an assessment of nutritional status. Albumin is a serum protein that is synthesized in the liver and therefore, may be reduced when liver function is impaired. The albumin level may also be affected by many other factors, including inflammation, infection, and metabolic stress. Prealbumin is a visceral protein that may be influenced by similar factors and may be falsely elevated in patients with renal failure. While albumin and prealbumin may be predictors of morbidity and mortality, these proteins are unreliable when predicting nutritional status. ${ }^{[27]}$

Calorimetry is the most accurate means to measure nutritional needs; however, it is often not readily available. When considering dietary recommendations for liver disease, caloric need should be individualized. Patients with liver disease may either be hypermetabolic or hypometabolic. Patients with stable, compensated cirrhosis are estimated to require $25-35 \mathrm{kcal} / \mathrm{kg}$ per day. Patients who are malnourished are estimated to require $30-40 \mathrm{kcal} / \mathrm{kg}$ per day. ${ }^{[7]} \mathrm{A}$ decrease of $500-1000$ calories daily may be considered for a patient who is obese with non-alcoholic fatty liver disease (NAFLD) in order to promote a gradual weight loss. [28] Protein needs may be monitored in patients with encephalopathy, but protein should not be restricted. Patients with cirrhosis require 1.0$1.5 \mathrm{~g} / \mathrm{kg}$ of protein per day to prevent muscle catabolism. ${ }^{[7]}$ There is no real evidence that protein restriction is beneficial for encephalopathy. Non-meat sources containing BCAAs should be incorporated into the diet of patients with encephalopathy since normalization of BCAAs promotes protein synthesis and reduces plasma ammonia, which promotes protein anabolism. ${ }^{[29]}$ Carbohydrates and small, frequent meals 


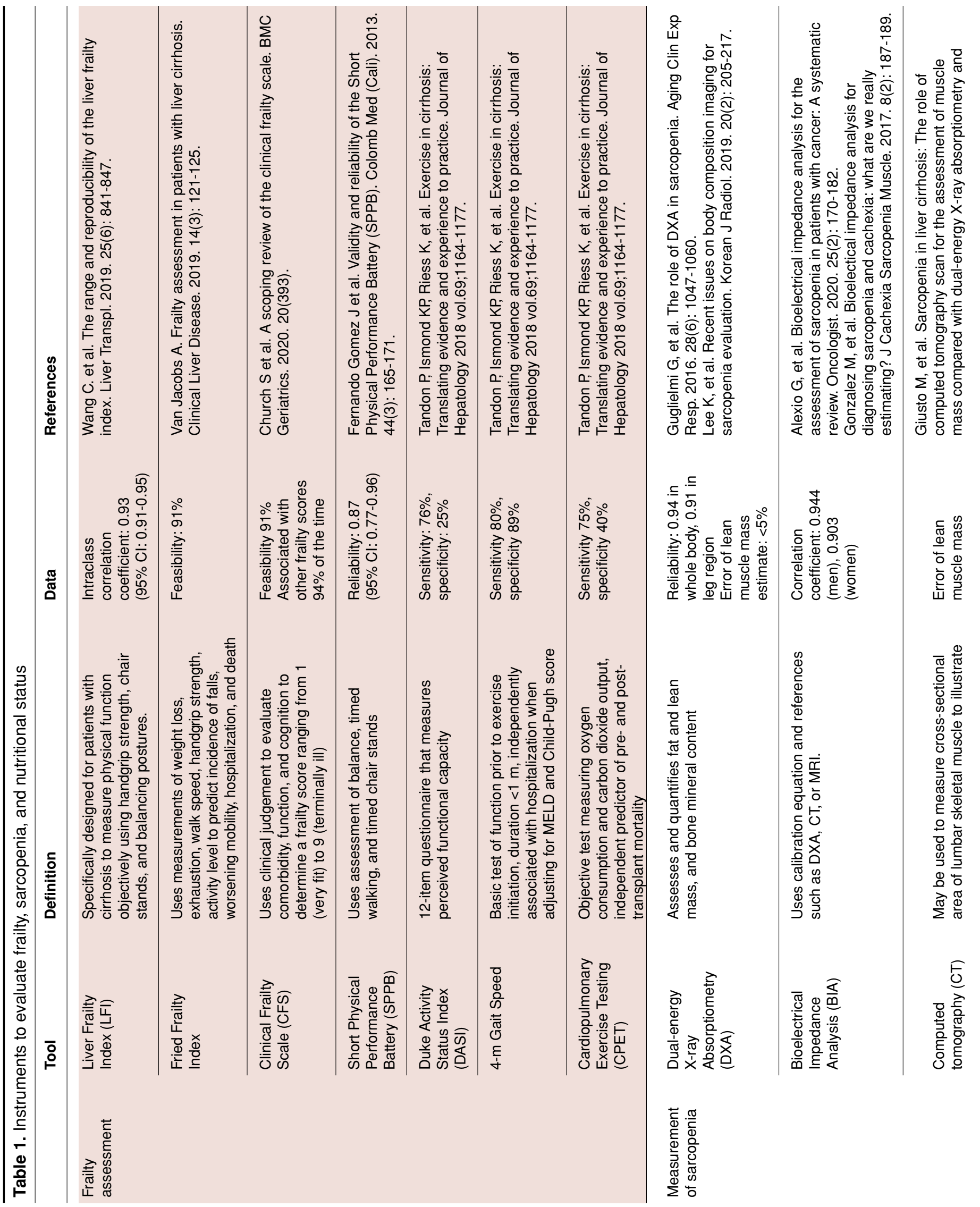




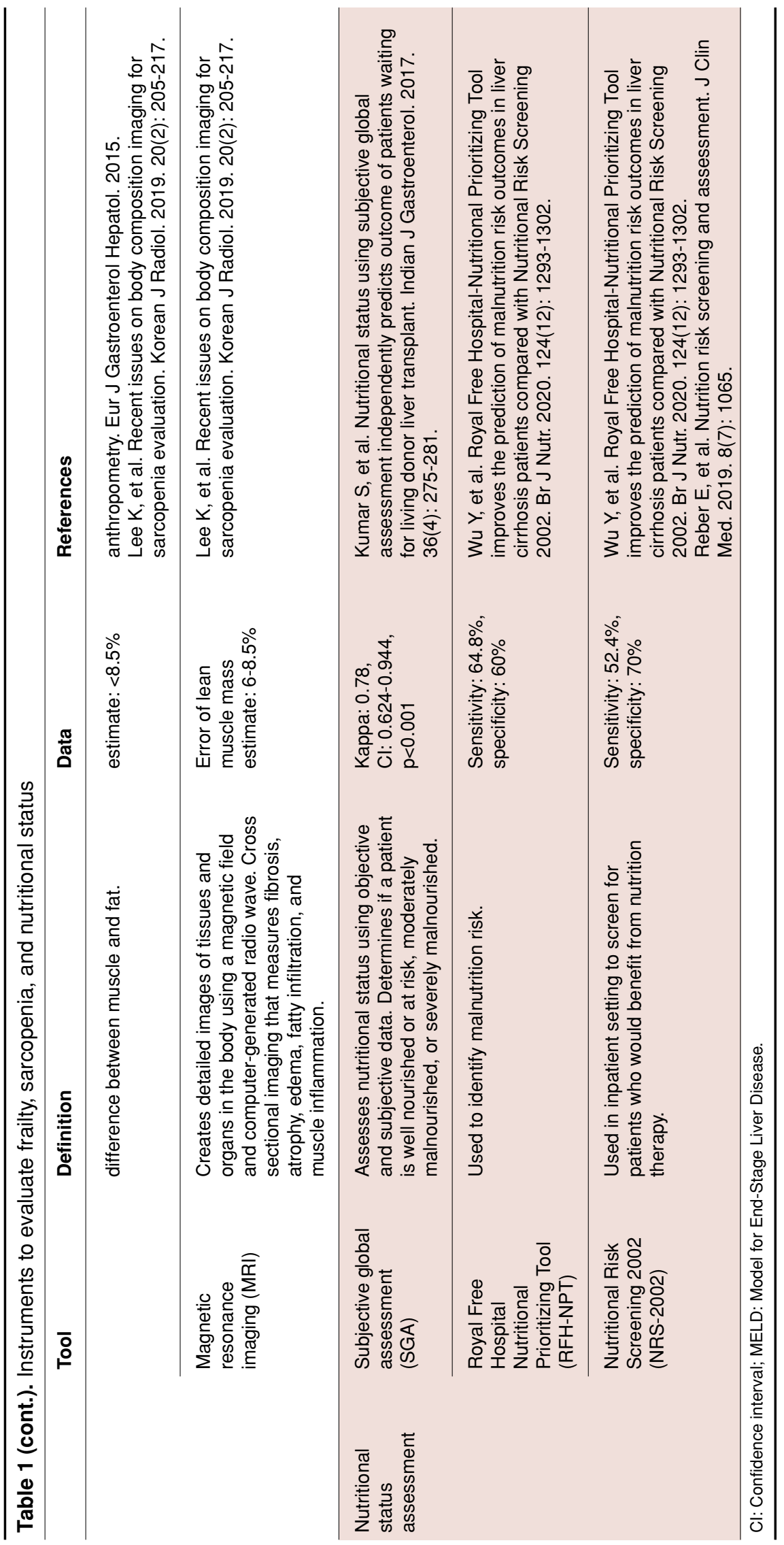

should be encouraged to prevent hypoglycemia. Late-evening snack consumption has been reported to improve liver function reserve and may reduce sarcopenia, which contributes to improved quality of life. ${ }^{[30]}$ Fat should only be limited if the patient is cholestatic, and this intervention should be temporary. Medium-chain triglycerides should be recommended with a fat-restricted diet to help prevent unintentional weight loss. ${ }^{[3]]}$ Potassium intake may need to be adjusted depending on the type of diuretic therapy applied. Some patients may need to limit intake of potassium if they use a potassium-sparing diuretic, such as spironolactone, or intake may need to be increased if they use a loop diuretic, such as furosemide. Sodium restrictions are indicated for most patients with cirrhosis to control ascites and edema. Fluid restriction may be indicated in some cases, particularly if the patient is hyponatremic. Early nutrition education and interventions are important to the effort to prevent or improve malnutrition. Priorities for these patients include education on adequate caloric and protein consumption, meal composition and timing, and protein sources, with an emphasis on more BCAArich options. ${ }^{[32]}$

Dietary interventions can help to optimize a patient's nutritional status. The first step should be to expand and improve the variety of dietary selections, if possible. Patients may also benefit from oral nutrition supplements. It is important to consider the taste and realistic compliance with a supplement regime. Cost is also a factor, as many insurance plans do not include oral nutrition supplements. Fluid and dietary restrictions also need to be factored in when recommending oral nutrition supplements. Nutrition education should also be provided by a registered dietitian to review the intricacies of intake, the types of foods to choose, and ways to increase calories and protein with dietary restrictions. This is important when a sodiumor potassium-restricted diet is advised, as patients may be unaware of the content in some food items.

In cirrhotic patients who are malnourished, enteral nutrition may improve survival by improving nutritional status, liver function, and decreasing complications. ${ }^{[33]}$ Nasogastric feeding tubes should be preferred to percutaneous endoscopic gastrostomy tubes when feasible, due to the increased risk of mortality, particularly in patients with ascites. ${ }^{[32]}$ Enteral nutrition helps to maintain gut wall function and gut wall integrity, and also helps to prevent bacterial translocation. Enteral nutrition also has a lower risk of infection. ${ }^{[34]}$ Patient resistance can be a barrier to initiating enteral nutrition support; however, this resistance can often be overcome with education, answering questions, and addressing concerns. Another perceived barrier is the presence of esophageal varices, due to concern about 
variceal bleeding; however, the risk of bleeding is low and therefore enteral nutrition may still be indicated. Careful placement of feeding tubes and monitoring is essential to reduce risk of variceal bleeding. ${ }^{[35]}$ If the patient requires a short-term rehabilitation stay, this may also present a difficulty since many facilities will not accept patients with nasal feeding tubes. A search for appropriate facilities should be initiated early in the hospital admission period. However, if the patient will be going home, many home care agencies have specialized nurses and dietitians to assist patients with enteral nutrition. There is also often better insurance coverage for tube feedings than oral nutrition supplements in the US.

Total parenteral nutrition (TPN) may also need to be considered in patients with cirrhosis to improve nutritional status. ${ }^{[36]}$ Parenteral nutrition is indicated for patients who cannot be fed orally. ${ }^{[36]}$ This applies to patients with an impaired gastrointestinal tract who have had a nothing per oral (NPO) restriction for >7-10 days, or if malnourished or critically ill and NPO for $>3-5$ days. ${ }^{[37]}$ When indicated, parenteral nutrition can improve nutritional status and liver function in patients with cirrhosis. ${ }^{[36]}$ A lipid emulsion that provides essential and non-essential fatty acids (SMOF emulsion) can have a positive impact on liver function in comparison to lipids providing only one type of fat (i.e., intralipid only provides soybean oil). An SMOF emulsion contains coconut (30\%), soybean $(30 \%)$, olive $(25 \%)$, and fish $(15 \%)$ oil. ${ }^{[38]}$ It is important to take care to prevent infectious complications associated with central line placement and maintenance.

Management of sodium, potassium, and fluid levels in patients with cirrhosis is critical. Hyponatremia may occur as a result of hemodynamic changes and secondary adaptations that lead to an inability to excrete water. The ability to expel water becomes more impaired as liver disease progresses. Clinical manifestations of hyponatremia can include confusion, fatigue, dizziness, nausea, and weakness. However, drastic correction and a high rate of sodium excretion is associated with urinary potassium losses. Treatment of hypokalemia may include discontinuing certain medications including diuretics, beta and alpha blockers, and antihypertensives. Increased serum sodium may help to correct hypokalemia and achieve a better balance. Diuretic adjustment, plasma expansion, and potentially the use of hypertonic saline may need to be considered when treating hyponatremia. Hemodialysis may be an option for patients who have severe renal impairment. Fluid restriction should be avoided, if possible, since in order to be effective, fluid restriction would have to be less than the total urine output. Most patients will not tolerate less than 1-1.5 liters per day. Moderate fluid restrictions may be helpful in patients who drink large quantities of fluid. ${ }^{[39]}$

Identifying and correcting micronutrient deficiencies is also important in ESLD as these patients are at greater risk. ${ }^{[40]}$ Cholestasis and malabsorption can lead to a deficiency of the fat-soluble vitamins A, D, E, and K. Zinc deficiency and decreased ability of the liver to store vitamin A may also contribute to vitamin A deficiency, and impaired hydroxylation and reduced synthesis may contribute to vitamin D deficiency.

In addition, thiamine deficiency may occur as a result of alcoholic liver disease due to malabsorption and poor diet quality. Thiamine deficiency may also be a consequence of diuretic medication use. Reduced liver storage capacity, malabsorption, and renal reabsorption may also lead to a folic acid deficiency. Pyridoxine and cobalamin deficiencies may be caused by poor diet quality and impaired metabolism. A poor diet, physiological stressors, and dialysis may also create a vitamin $\mathrm{C}$ deficiency, and altered protein metabolism, decreased production of al- bumin, urinary losses, muscle catabolism, malabsorption, and losses from portosystemic shunt can result in a zinc deficiency. Furthermore, a copper deficiency is more common in patients with alcoholic liver disease and NAFLD, or may be an outcome of excessive zinc supplementation. ${ }^{[40]}$ Patients with alcoholic liver disease or HCC may also be at risk for a selenium deficiency. Micronutrient deficiencies should be assessed carefully in patients with liver disease and supplements should be prescribed as needed.

Other conditions that may impact nutritional status should also be evaluated, such as bacterial overgrowth and testosterone deficiency. Malnutrition is more likely in patients with small intestinal bacterial overgrowth. ${ }^{[41]}$ Testosterone deficiency may be associated with sarcopenia in males with liver disease. ${ }^{[42]}$

Refeeding syndrome is another concern in patients with cirrhosis. Refeeding syndrome occurs with severe fluid and electrolyte shifts due to rapid introduction of nutrition after prolonged fasting or undernutrition. ${ }^{[43]}$ When the body is in a state of starvation, insulin levels are low and glucagon levels are elevated. This condition leads to protein catabolism as a source of energy. Starvation creates a depletion intracellular minerals, however, serum levels remain normal. During refeeding, insulin levels rise, and potassium, phosphorus, and magnesium are drawn into the cells, which leads to low levels in the serum. Clinical manifestations can include hypophosphatemia, hypokalemia, hypomagnesemia, hyperglycemia, and fluid intolerance. ${ }^{[4]}$ A patient is at risk for refeeding syndrome if they meet one or more of these criteria: 1) a $\mathrm{BMI}<16 \mathrm{~kg} / \mathrm{m}^{2}$, 2) $15 \%$ unintentional weight loss in the past 3-6 months, 3) little or no nutritional intake for $>10$ days, or 4) low levels of potassium, magnesium, and phosphorus prior to feeding; or if they meet two or more of the following criteria: 1) a $\mathrm{BMI}<18.5 \mathrm{~kg} / \mathrm{m}^{2}, 2$ ) unintentional weight loss $>10 \%$ in the past 3-6 months, 3) little or no nutritional intake for 5 days, 4) a history of alcohol abuse, or 5) use of drugs, including insulin, chemotherapy, antacids, or diuretics. ${ }^{[45]}$ When initiating enteral feeding, laboratory values should be monitored frequently with repletion of electrolytes provided as needed. Feeds should be titrated up very slowly. Subsequent tube feedings should be individualized according to patient tolerance. Patients should be instructed to report any symptoms of intolerance, such as nausea, vomiting, or diarrhea. However, enteral nutrition is safe and well tolerated by most patients. Calorically dense formulas $(1.5-2$ calories $/ \mathrm{mL})$ may be valuable due to the lower volume needed to meet nutritional requirements. Patients with steatorrhea may benefit from a formula with a greater quantity of medium chain triglycerides. ${ }^{[46]}$ Mechanical issues may also occur, such as nasojejunal tube dislodgement or clogging. A nasal bridle should be considered, as it has been shown to significantly reduce dislodgement, although there is an increased risk of skin complications. ${ }^{[4]}$

We implemented a nutrition protocol at our center to assess and treat malnutrition in patients with ESLD. This care algorithm may serve as a prototype for others who wish to develop a standard of practice (Fig. 1). The initial step is to provide nutrition education to patients to assist them with meeting their nutritional needs while following any necessary dietary restrictions, such as a low-sodium diet. The presence of malnutrition is assessed using a SGA. Oral nutrition supplements are recommended if needed. If a patient is subsequently unable to meet their nutritional needs, then enteral nutrition support is initiated. Patients requiring enteral nutrition support are hospitalized to ensure tolerance and prevent refeeding syndrome. The key is reassessment at short intervals and implementation of rapid adjustments as needed. 


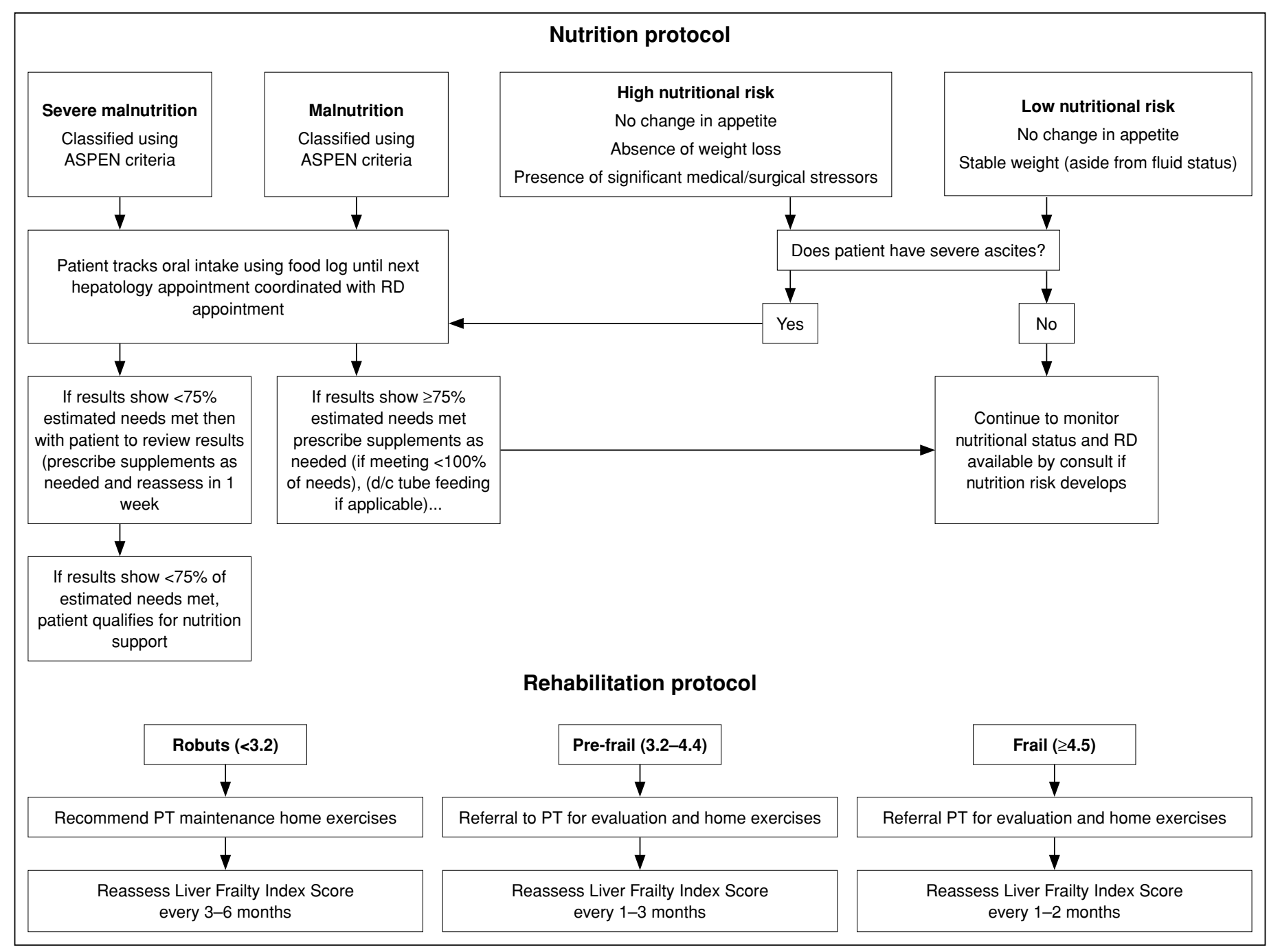

Figure 1. Pre-liver transplant frailty treatment algorithm.

ASPEN: American Society for Parenteral and Enteral Nutrition; PT: Physical therapy; RD: Registered dietician.

\section{Key Points}

- Nutritional assessment includes both objective and subjective data. Formalized assessment tools are available. Use of BMI may be limited by fluid status.

- Priorities include education re: adequate caloric and protein intake with emphasis on BCAAs, meal composition, and timing.

- Frequent assessment of caloric intake and weight trends with early addition of oral supplements is essential.

- Since enteral nutrition improves survival, rapid initiation may become appropriate in frail patients not achieving caloric needs.

- Enteral nutrition is safe and well tolerated by most patients, though they are at risk for refeeding syndrome. Titration of tube feeding should be slow and include electrolyte monitoring.

- TPN should be considered when nutrition cannot be provided through the gut.

- Overall fluid and electrolyte management in patients with cirrhosis is critical.

- Micronutrient deficiencies are common, and repletion is important.

\section{Physical Debility in ESLD: Rehabilitation Interventions}

It is essential to pair nutrition protocols with physical rehabilitation protocols that incorporate exercise prescriptions targeted to treating physical debility. Supervised exercise programs may not be an option for some patients; however, home-based programs can be helpful for many patients. ${ }^{[48]}$ Frailty and sarcopenia may lead to poor outcomes in patients who undergo LT. ${ }^{[49]}$ Exercise programs have been shown to improve functional capacity and may help to improve metabolic diseases, which are common after LT. ${ }^{[50]}$

Like the nutritional assessment, the assessment of physical function draws from both objective and subjective data. The Karnofsky performance status (KPS), which assess a patient's ability to perform activities of daily living (e.g., bathing, toileting, transferring, ambulating, eating, and dressing), has been used for over 70 years to assess patients before and after LT. It is an established independent predictor of graft and patient survival, and is required for United Network for Organ Sharing reporting of risk adjustment in outcomes. ${ }^{[51]}$ As noted previously, formal frailty assessments, such as the LFI, should be performed as well. Physical debility increases as patient condition declines from robust $(\mathrm{LFI}<3.2)$ to pre-frail (LFI 3.2-4.5) to frail $(>4.5)$. 
Cirrhotic patients spend much of their time in a sedentary state ${ }^{[49]}$ however, it has been demonstrated that exercise improves aerobic power, endurance, muscle mass, strength, health-related quality of life, and even portal hypertension in this population. Professional societies, such as the American College of Sports Medicine, the AASLD, and the AST, endorse the importance of physical rehabilitation/exercise prescriptions for cirrhosis patients. ${ }^{[49]}$

A University of California-San Francisco group used the LFI to categorize patients as robust, pre-frail, or frail and developed a standard protocol to manage physical debility with specified rehabilitation services. If a patient has severe frailty, a rehabilitation program is prescribed, which may include inpatient rehabilitation for 2-4 weeks. Temporary waitlist inactivation is considered. Frailty is reassessed every 2-6 weeks, with a plan to proceed with a transplant if frailty is successfully reversed. If a patient has moderate frailty, a home-based exercise program is prescribed. These patients are closely monitored while on the waitlist and frailty is evaluated after $4-12$ weeks. If there is no further deterioration, then they may proceed with LT. For patients with mild or no significant frailty, the goal is to exercise for a minimum of 150 minutes per week. Patients are instructed to build up to this goal slowly; for example, they may start with 10 minutes of exercise 2-3 times daily. There is no change to waitlist management during this time. Frailty is reassessed every 12 weeks until LT. ${ }^{[52]}$ A study conducted at the University of Michigan Health System found that frailty impacted quality of life in LT candidates and the authors noted a plan to implement an intervention program in the future. ${ }^{[53]}$ Other centers also use similar approaches to assess and manage frailty in patients with ESLD who are awaiting LT; however, this is not yet universal.

Frailty assessment and the initiation of rehabilitation protocols in the outpatient setting is ideal, but not always possible, since patients are referred at different stages of disease, may be first encountered by transplant teams in the inpatient setting, or require frequent hospitalization. Regardless of the setting, it is important to recognize that reliance on physical therapy sessions alone (for example 1 hour, 3 times per week) is not sufficient and must be incorporated as just one component to increase the overall activity level with the assistance of a caregiver at home or a multidisciplinary team as an inpatient. In the hospital setting, standardized assessments, such as the Activity Measure for Post-Acute Care, ${ }^{[54]}$ the Inpatient Mobility Short Form, also called the "6-Clicks," ${ }^{[55]}$ and the Johns Hopkins Highest Level of Mobility, have proven validity and interrater reliability among a multidisciplinary team for the measurement of patient mobility and physical functioning in both the intensive care unit and general medicine wards. ${ }^{[56]}$ Such measures can predict the acute care hospital discharge destination. Implementation of mobility initiatives have been shown to improve mobility and reduce the length of stay and hospital readmissions. ${ }^{[57]}$ It is likely that this will be generalizable to the ESLD population; however, this will need to be formally studied.

In a beautiful recent review, Tandon et al. ${ }^{[49]}$ summarized current data related to exercise and cirrhosis, noted barriers to implementation, and provided concrete recommendations and a readily accessible toolkit of resources to overcome obstacles and propel healthcare teams to an active role in addressing frailty. They presented a three-step process of: 1) screening to minimize exercise-related adverse events, 2) baseline physical capacity assessment, and 3) exercise programming with subsequent monitoring. Details of safety assessment are provided with the tenet of "start low and go slow." The authors highlighted the value of non-exercise activity thermogenesis (NEAT), which involves continu- ous and vital low-intensity movements that can be achieved by taking advantage of opportunities for physical activity within day-to-day routines and increase overall activity levels. They define formal exercise as distinct from physical activity in that it is planned and performed on a repeated basis over an extended period to improve fitness, performance, and health. It is recommended that training prescriptions should follow the FITT principles: frequency, intensity, time, and type of exercise. Resources provided include sample questions for motivational interviewing, sample exercise prescriptions, and instructional exercise videos.

New models of care delivery aimed at overcoming barriers such as resource limitation/accessibility are a current focus. This includes wearable activity monitors, video streaming, and smart phone applications. ${ }^{[49]}$ The Exercise and Liver FITness (EL-FIT) smartphone application developed by hepatologist Andres Duarte-Rojo is an example of this kind of innovation. EL-FIT provides an exercise training program for patients with liver disease and tracks participant activity via Fitbit (Fitbit Inc., San Francisco, CA, USA). The results of a feasibility study in 28 participants revealed that the level of training assigned by the EL-FIT application agreed with that of a physical therapist in $89 \%$ of cases. The study participants could interact with several features, such as videos, perceived exertion, and gamification/ motivational elements. The findings indicated that $35 \%$ of the participants demonstrated a significant increase in physical performance. The authors noted that the participants emphasized their interest in having choices for exercise engagement and appreciation of the sense of community the EL-FIT app generated ${ }^{[58]}$ Further data collection with an evaluation of the impact on frailty is ongoing, and real-world use at other institutions will be valuable.

In our program, we approached the issue of frailty in the LT population in a programmatic fashion using Quality Assurance and Performance Improvement meetings as a platform to develop a multidisciplinary approach and protocol to treat physical debility that would pair with our pre-existing nutrition protocol (Fig. 1). Our aim is to address frailty and thus reduce waitlist mortality and optimize transplant outcomes. We have established a formal collaboration with the rehabilitation services department. An educational slide show and webinar related to the pathophysiology of ESLD, unique characteristics of this patient population as far as rehabilitation services (i.e., sarcopenia, volume overload, hepatic encephalopathy, varices, use of beta blockers, thrombocytopenia), and the significance of frailty on clinical outcomes was developed and delivered to the rehabilitation medicine department/physical therapy (PT) providers. A formal referral process for rehabilitation services was also created. Formal frailty testing with the LFI was incorporated into outpatient visits at the transplantation center. Patients who are robust are provided with maintenance exercises to be performed at home and a frailty assessment every 3-6 months. Patients who are classified as prefrail are referred for a formal PT evaluation with subsequent outpatient appointments and prescribed home exercises as well as a frailty evaluation every 2-3 months. Patients who are frail are referred for a formal PT evaluation with subsequent outpatient appointments and home exercises with a frailty assessment every 1-2 months. We plan to incorporate use technology such as the EL-FIT application and we are working to establish a mobility initiative in our hospital-based liver unit.

\section{Key Points}

- Physical debility and sarcopenia are common in ESLD and contribute to frailty. Validated assessment tools are available and should be utilized. 
- Priorities include education re: importance of increased physical activity and tools and resources for patients/families.

- A multidisciplinary approach and partnership with rehabilitation teams is essential to achieve goals.

- Guidance re: opportunities to increase NEAT via adjustments in day-to-day routines should be provided.

- Formal exercise prescriptions, including definition of frequency, intensity, time, and type of exercise should be incorporated into treatment plans.

- Resource limitations and barriers to implementation may be overcome with the incorporation of home-based regimens and utilization of available technologies.

\section{Summary and Conclusions}

Careful assessment of frailty and early intervention targeting malnutrition and physical debility for patients with ESLD awaiting LT is essential. The development of standards of practice with formalized treatment protocols incorporating frequent reassessment and rapid increments in nutrition support via oral supplements, enteral feeding, or TPN if required, and rehabilitation programming incorporating NEAT with formal exercise prescriptions and PT sessions via a multidisciplinary team are essential steps. Nutrition and exercise regimens should be crafted according to the degree of sarcopenia and debility. Further clinical research examining the impact of such programming on frailty and clinical outcomes is necessary. Ongoing study of the innovative use of technology to aid in treatment and real-world applicability is also required, as well as study of the impact of mobility initiatives in the acute care setting.

Peer-review: Externally peer-reviewed.

Author Contributions: Concept - AML, EM, SE; Design - AML, SE; Supervision - AML, SE; Literature Search - AML, EM, SE; Writing - AML, EM, SE; Critical Reviews - AML, SE.

Conflict of Interest: The authors have no conflict of interest to declare.

Financial Disclosure: The authors declared that this study has received no financial support.

\section{References}

1. Clegg A, Young J, Iliffe S, Rikkert MO, Rockwood K. Frailty in elderly people. Lancet 2013;381(9868):752-762. [CrossRef]

2. Lai JC, Rahimi RS, Verna EC, Kappus MR, Dunn MA, McAdams-DeMarco M, et al. Frailty Associated With Waitlist Mortality Independent of Ascites and Hepatic Encephalopathy in a Multicenter Study. Gastroenterology 2019;156(6):1675-1682. [CrossRef]

3. Tandon P, Reddy KR, O’Leary JG, Garcia-Tsao G, Abraldes JG, Wong F, et al; North American Consortium for the Study of End-Stage Liver Disease. A Karnofsky performance status-based score predicts death after hospital discharge in patients with cirrhosis. Hepatology 2017;65(1):217-224.

4. Meyer F, Bannert K, Wiese M, Esau S, Sautter LF, Ehlers L, et al. Molecular Mechanism Contributing to Malnutrition and Sarcopenia in Patients with Liver Cirrhosis. Int J Mol Sci 2020;21(15):5357. [CrossRef]

5. Aby ES, Saab S. Frailty, Sarcopenia, and Malnutrition in Cirrhotic Patients. Clin Liver Dis 2019;23(4):589-605. [CrossRef]

6. Lai JC, Feng S, Terrault NA, Lizaola B, Hayssen H, Covinsky K. Frailty predicts waitlist mortality in liver transplant candidates. Am J Transplant 2014;14(8):1870-1879. [CrossRef]

7. Johnson TM, Overgard EB, Cohen AE, DiBaise JK. Nutrition assessment and management in advanced liver disease. Nutr Clin Pract 2013;28(1):15-29.

8. Campos-Varela I, Gómez-Gavara C, Augustin S. Recommendations and guidance on nutritional supplementation in the liver transplant setting. Transplantation 2021 Mar 8. doi: 10.1097/TP.0000000000003736. [Epub ahead of print]

9. Ebadi M, Bhanji RA, Mazurak VC, Montano-Loza AJ. Sarcopenia in cirrhosis: from pathogenesis to interventions. J Gastroenterol 2019;54(10):845859. [CrossRef]

10. Dolgin NH, Martins PN, Movahedi B, Lapane KL, Anderson FA, Bozorgzadeh A. Functional status predicts postoperative mortality after liver transplantation. Clin Transplant 2016;30(11):1403-1410. [CrossRef]

11. Durand F, Buyse S, Francoz C, Laouénan C, Bruno O, Belghiti J, et al. Prognostic value of muscle atrophy in cirrhosis using psoas muscle thickness on computed tomography. J Hepatol 2014;60(6):1151-1157. [CrossRef]

12. Englesbe MJ, Patel SP, He K, Lynch RJ, Schaubel DE, Harbaugh C, et al. Sarcopenia and mortality after liver transplantation. J Am Coll Surg 2010;211(2):271-278. [CrossRef]

13. Dodds R, Sayer AA. Sarcopenia and frailty: new challenges for clinical practice. Clin Med (Lond) 2016;16(5):455-458. [CrossRef]

14. Lai JC, Covinsky KE, Dodge JL, Boscardin WJ, Segev DL, Roberts JP, et al. Development of a novel frailty index to predict mortality in patients with end-stage liver disease. Hepatology 2017;66(2):564-574. [CrossRef]

15. Wang CW, Lebsack A, Chau S, Lai JC. The Range and Reproducibility of the Liver Frailty Index. Liver Transpl 2019;25(6):841-847. [CrossRef]

16. Wiesner R, Edwards E, Freeman R, Harper A, Kim R, Kamath P, et al; United Network for Organ Sharing Liver Disease Severity Score Committee. Model for end-stage liver disease (MELD) and allocation of donor livers. Gastroenterology 2003;124(1):91-96. [CrossRef]

17. Merion RM, Schaubel DE, Dykstra DM, Freeman RB, Port FK, Wolfe RA. The survival benefit of liver transplantation. Am J Transplant 2005;5(2):307313. [CrossRef]

18. Biggins SW, Kim WR, Terrault NA, Saab S, Balan V, Schiano T, et al. Evidence-based incorporation of serum sodium concentration into MELD. Gastroenterology 2006;130(6):1652-1660. [CrossRef]

19. Lai J. When is transplant a futile option and enough is enough. Conference presentation in The Liver Meeting AASLD; 2017, Oct 20-24; Washington D.C.: United States; 2017.

20. Salim TI, Nestlerode LC, Lucatorto EL, Wasserman TL, Din HA, Landsittel DP, et al. Frailty as Tested by Gait Speed Is a Risk Factor for Liver Transplant Respiratory Complications. Am J Gastroenterol 2020;115(6):859-866.

21. Lai JC, Segev DL, McCulloch CE, Covinsky KE, Dodge JL, Feng S. Physical frailty after liver transplantation. Am J Transplant 2018;18(8):19861994. [CrossRef]

22. Thasneem S, Yedu Krishnan SS, Roshni PR, Shine Sadasivan. A review of frailty in patients with liver cirrhosis and it's management. Journal of Liver 2020;9(2):1-7.

23. Lai J, Sonneday CJ. Emerging Trends Symposium. Conference Preceedings in The Liver Meeting AASLD; 2017, Oct 20-24; Washington D.C.: United States; 2017.

24. Kobashigawa J, Dadhania D, Bhorade S, Adey D, Berger J, Bhat G, et al. Report from the American Society of Transplantation on frailty in solid organ transplantation. Am J Transplant 2019;19(4):984-994. [CrossRef]

25. Ciocîrlan M, Cazan AR, Barbu M, Mănuc M, Diculescu M, Ciocîrlan M. Subjective Global Assessment and Handgrip Strength as Predictive Factors in Patients with Liver Cirrhosis. Gastroenterol Res Pract 2017;2017:8348390. [CrossRef]

26. Wu Y, Zhu Y, Feng Y, Wang R, Yao N, Zhang M, et al. Royal Free HospitalNutritional Prioritizing Tool improves the prediction of malnutrition risk outcomes in liver cirrhosis patients compared with Nutritional Risk Screening 2002. Br J Nutr 2020;124(12):1293-1302. [CrossRef]

27. Parris C. Serum proteins as markers of nutrition: what are we treating? $\mathrm{Nu}-$ trition Issues in Gastroenterology 2006;43:46-64. 
28. Rusu E, Enache G, Jinga M, Dragut R, Nan R, Popescu H, et al. Medical nutrition therapy in non-alcoholic fatty liver disease--a review of literature. J Med Life 2015;8(3):258-262.

29. Cabral CM, Burns DL. Low-protein diets for hepatic encephalopathy debunked: let them eat steak. Nutr Clin Pract 2011;26(2):155-159. [CrossRef]

30. Chen CJ, Wang LC, Kuo HT, Fang YC, Lee HF. Significant effects of late evening snack on liver functions in patients with liver cirrhosis: A meta-analysis of randomized controlled trials. J Gastroenterol Hepatol 2019;34(7):1143-1152. [CrossRef]

31. Send SR. Nutritional Management of Cholestasis. Clin Liver Dis (Hoboken) 2020;15(1):9-12. [CrossRef]

32. Moss O. Nutrition Priorities: Diet Recommendations in Liver Cirrhosis. Clin Liver Dis (Hoboken) 2019;14(4):146-148. [CrossRef]

33. Plauth M, Bernal W, Dasarathy S, Merli M, Plank LD, Schütz T, et al. ESPEN guideline on clinical nutrition in liver disease. Clin Nutr 2019;38(2):485521. [CrossRef]

34. Tsiaousi ET, Hatzitolios AI, Trygonis SK, Savopoulos CG. Malnutrition in end stage liver disease: recommendations and nutritional support. J Gastroenterol Hepatol 2008;23(4):527-533. [CrossRef]

35. Al-Obaid LN, Bazarbashi AN, Cohen ME, Kim J, Lei Y, Axelrad JE, et al. Enteric tube placement in patients with esophageal varices: Risks and predictors of postinsertion gastrointestinal bleeding. JGH Open 2019;4(2):256259. [CrossRef]

36. Plauth M, Cabré E, Campillo B, Kondrup J, Marchesini G, Schütz T, et al; ESPEN. ESPEN Guidelines on Parenteral Nutrition: hepatology. Clin Nutr 2009;28(4):436-444. [CrossRef]

37. McClave SA, Taylor BE, Martindale RG, Warren MM, Johnson DR, Braunschweig C, et al. Guidelines for the provision and assessment of nutrition support therapy in the Adult Critically Ill Patient: Society of Critical Care Medicine (SCCM) and American Society for Parenteral and Enteral Nutrition (A.S.P.E.N.). JPEN J Parenter Enteral Nutr 2016;40(2):159-211.

38. Leguina-Ruzzi AA, Ortiz R. Current evidence for the use of smoflipid ${ }^{\circledR}$ emulsion in critical care patients for parenteral nutrition. Crit Care Res Pract 2018;2018:6301293. [CrossRef]

39. Sterns R, Runyon B. Hyponatremia in patients with cirrhosis. Available at: https://www.uptodate.com/contents/hyponatremia-in-patients-with-cirrhosis. Accessed March 17, 2021.

40. Kozeniecki M, Ludke R, Kerner J, Patterson B. Micronutrients in liver disease: roles, risk factors for deficiency, and recommendations for supplementation. Nutr Clin Pract 2020;35(1):50-62. [CrossRef]

41. Yao J, Chang L, Yuan L, Duan Z. Nutrition status and small intestinal bacterial overgrowth in patients with virus-related cirrhosis. Asia Pac J Clin Nutr 2016;25(2):283-291.

42. Moctezuma-Velázquez C, Low G, Mourtzakis M, Ma M, Burak KW, Tandon $\mathrm{P}$, et al. Association between low testosterone levels and sarcopenia in cirrhosis: a cross-sectional study. Ann Hepatol. 2018 July - August 2018;17(4):615-623. [CrossRef]

43. Mehanna H, Nankivell PC, Moledina J, Travis J. Refeeding syndrome--awareness, prevention and management. Head Neck Oncol 2009;1:4.
44. Crook MA, Hally V, Panteli JV. The importance of the refeeding syndrome. Nutrition 2001;17(7-8):632-637. [CrossRef]

45. National Institute for Health and Clinical Excellence. Nutrition support for adults: oral nutrition support, enteral tube feeding and parenteral nutrition

Clinical guideline [CG32]. Available at: www.nice.org.uk/page.aspx ${ }_{0}=\operatorname{cg} 032$. Accessed March 17, 2021.

46. Rees Parish C. Nutrition for patients with hepatic failure. Nutrition Issues in Gastroenterology 2003;6:23-42.

47. Bechtold ML, Nguyen DL, Palmer LB, Kiraly LN, Martindale RG, McClave SA. Nasal bridles for securing nasoenteric tubes: a meta-analysis. Nutr Clin Pract 2014;29(5):667-671. [CrossRef]

48. Duarte-Rojo A, Ruiz-Margáin A, Montaño-Loza AJ, Macías-Rodríguez RU, Ferrando A, Kim WR. Exercise and physical activity for patients with end-stage liver disease: Improving functional status and sarcopenia while on the transplant waiting list. Liver Transpl 2018;24(1):122-139. [CrossRef]

49. Tandon P, Ismond KP, Riess K, Duarte-Rojo A, Al-Judaibi B, Dunn MA, et al. Exercise in cirrhosis: Translating evidence and experience to practice. $\mathrm{J}$ Hepatol 2018;69(5):1164-1177. [CrossRef]

50. Garcia AM, Veneroso CE, Soares DD, Lima AS, Correia MI. Effect of a physical exercise program on the functional capacity of liver transplant patients. Transplant Proc 2014;46(6):1807-1808. [CrossRef]

51. Thuluvath PJ, Thuluvath AJ, Savva Y. Karnofsky performance status before and after liver transplantation predicts graft and patient survival. J Hepatol 2018;69(4):818-825. [CrossRef]

52. Lai JC, Sonnenday CJ, Tapper EB, Duarte-Rojo A, Dunn MA, Bernal W, et als. Frailty in liver transplantation: An expert opinion statement from the American Society of Transplantation Liver and Intestinal Community of Practice. Am J Transplant 2019;19(7):1896-1906. [CrossRef]

53. Derck JE, Thelen AE, Cron DC, Friedman JF, Gerebics AD, Englesbe MJ, et al. Quality of life in liver transplant candidates: frailty is a better indicator than severity of liver disease. Transplantation 2015;99(2):340-344.

54. Hoyer EH, Young DL, Klein LM, Kreif J, Shumock K, Hiser S, et al. Toward a common language for measuring patient mobility in the hospital: reliability and construct validity of interprofessional mobility measures. Phys Ther 2018;98(2):133-142. [CrossRef]

55. Jette DU, Stilphen M, Ranganathan VK, Passek SD, Frost FS, Jette AM. AM-PAC "6-Clicks" functional assessment scores predict acute care hospital discharge destination. Phys Ther 2014;94(9):1252-1261. [CrossRef]

56. Hoyer EH, Friedman M, Lavezza A, Wagner-Kosmakos K, Lewis-Cherry $\mathrm{R}$, Skolnik JL, et al. Promoting mobility and reducing length of stay in hospitalized general medicine patients: A quality-improvement project. J Hosp Med 2016;11(5):341-347. [CrossRef]

57. Bergbower EAS, Herbst C, Cheng N, Aversano A, Pasqualini K, Hartline $\mathrm{C}$, et al. A novel early mobility bundle improves length of stay and rates of readmission among hospitalized general medicine patients. J Community Hosp Intern Med Perspect 2020;10(5):419-425. [CrossRef]

58. Duarte-Rojo A, Bloomer PM, Rogers RJ, Hassan MA, Dunn MA, Tevar $\mathrm{AD}$, et al. Introducing EL-FIT (Exercise and Liver FITness): A Smartphone App to Prehabilitate and Monitor Liver Transplant Candidates. Liver Transpl 2021;27(4):502-512. [CrossRef] 